\title{
ANDERS ZUHÖREN
}

\section{Tribe 8s ästhetische Praktiken: «Disidentification» und queere Klassenpolitiken ${ }^{1}$}

1 Ich möchte zunächst Lia Becker herzlich danken für die produktiven Diskussionen über den gemeinsam entwickelten Begriff «Klasse mit Differenz", der in diesem Beitrag noch eine prominente Rolle spielen wird, und für ihre immense Unterstützung meiner Arbeit.

2 Queercore. How to Punk a Revolution, Regie: Yony Leyser, D 2017.

3 Er_sie trat damals noch unter dem Namen Lynn Breedlove auf, inzwischen hat er Namen und Pronomen verändert.

4 Vgl. Atlanta Ina Beyer: Tribe 8, in: Jonas Engelmann (Hg.): Damaged Goods. 150 Einträge in die Punkgeschichte, Mainz 2016, 278-280, hier 279.

5 Vgl. Lisa Duggan: Sex Wars. Sexual Dissent and Political Culture, New York 2006 [1996], 1; Volker Woltersdorff alias Lore Logorrhöe: Queer Theory und Queer Politics, in: Utopie Kreativ, Nr. 156, 2003, 914-923, hier $914 \mathrm{f}$.

6 Die Abkürzung steht für die sexuellen Praktiken: Bondage and Discipline (Fesselung und Disziplin), Dominance and Submission (Dominanz und Unterwerfung), Sadism and Masochism (S/M).

7 Elisabeth Joyce: Postfeminism as Recombinant Fragment, in: Birgit Haas (Hg.): Der postfeministische Diskurs, Würzburg 2006, 105-126, hier 113 .
Ende 2017 kam mit Yony Leysers Queercore. How to Punk A Revolution ${ }^{2}$ eine Dokumentation in die Kinos, die die Geschichte von Queercore, einer queeren Strömung im Punk, beleuchtet. In Leysers Film wird auch Lynnee Breedlove interviewt, Sänger bzw. damals noch Sängerin ${ }^{3}$ der Band Tribe 8, die sich 1990 in San Francisco gründete und dort 2005 wieder auflöste. Die Band wird nicht nur Queercore, sondern auch der bekannteren, feministischen Punkbewegung der Riot Grrrls zugeordnet. Anfang der r99oer Jahre artikulieren diese neuen Punkpolitiken ihren enormen Frust auf die gesellschaftlichen Zustände. Im vorangegangenen Jahrzehnt war die christliche Rechte in den USA erstarkt und sorgte mit ihrer Anti-Abtreibungs-Politik, der Stimmungsmache gegen Aidserkrankte, Homosexuelle und sozial Marginalisierte für einen politischen und kulturellen Backlash. ${ }^{4}$

Tribe 8 lösten mit ihren sexpositiven, oft spektakulären Entwürfen viele Kontroversen auch innerhalb lesbisch-feministischer Szenen aus. Seit etwa Mitte der I970er Jahre hatten Themen wie Pornografie, Sadomasochismus oder Penetration zu erbittert geführten innerfeministischen Grabenkämpfen, sogenannten Sex Wars, geführt. ${ }^{5}$ Die Band bezog gegenüber BDSM ${ }^{6}$-Praktiken eine eindeutig positive Haltung. Die meisten Mitglieder identifizierten sich mit dem eher maskulinen lesbischen Genderentwurf der butch; Leslie Mah, eine der Gitarrist_innen, bezeichnete sich als feminine femme-Lesbe.

Elisabeth Joyce beschreibt die Zusammensetzung von Tribe 8 als «racial mixture of women - African American, Asian American, Euro-American - of middle class and blue collar background with little or no higher education.» ${ }^{7}$ Aus Interviews mit Bandmitgliedern entnehme ich, dass sie vor allem aus der unteren Mittel- und der Arbeiter_innenklasse kommen. Ihre künstlerischen Praktiken scheinen an der Oberfläche jedoch zunächst wenig über 〈Klasse〉 preiszugeben. Dies ist in queerfeministischen Politiken eher die Regel: Themen um Gender und Sexualität rücken in den Mittelpunkt, 
Klassenidentifikationen dagegen werden ausgeblendet. So werden auch die ästhetischen Praktiken queerer Subkultur vorrangig als Aushandlungen um Gender- und sexuelle Praktiken, Identitäten und Politiken gelesen und analysiert. Ich möchte eine andere Lesart vorschlagen.

Ich gehe davon aus, dass in queerer Subkultur eine komplexe Artikulation/Des-Artikulation von Klasse stattfindet. Um diese aufspüren zu können, ist es nötig, die in der politischen wie wissenschaftlichen Debatte häufig reproduzierte, aber unproduktive Dichotomie zwischen Identitäts- und Klassenpolitiken aufzulösen, die den Blick auf die vielfachen und widersprüchlichen Verschränkungen beider verstellt. Durch ein «Re-Framing», ${ }^{8}$ in dem ich kritisch an die Re-Artikulation des Klassenbegriffs in den britischen Cultural Studies und in queerer Theorie ${ }^{9}$ anknüpfe und in dem ich den Blick auf die im Zuge der Aidskrise entstehenden neuen queeren Politiken der Wut ${ }^{10}$ richte, will ich die Spuren der zugleich abwesenden und anwesenden $<$ Klasse $>$ in den ästhetischen Praktiken von Tribe 8 freilegen.

Durch ein doppeltes Lesen ausgewählter Arbeiten der Band (Songtexte, Gesangstechniken und Kontroversen um einen Auftritt) werden - so meine These - Praktiken der «disidentification»" ${ }^{11}$ mit lesbisch-feministischer Bewegung und Subkultur deutlich, die Klassendifferenzen wahrnehmbar machen und einen ermächtigenden Umgang mit Gewalterfahrungen und Traumata ermöglichen. Die Verortung der ästhetischen Praktiken von Tribe 8 im Kontext neuer queerer Politiken der Wut zeigt, wie sie zur Erweiterung von Allianzen marginalisierter Gruppen beitragen. Durch die Perspektivverschiebung weg von der dichotomen Gegenüberstellung von Klassenidentität vs. queerer Identität hin zum Konzept «Klasse mit queer-feministischer Differenz» können das transformatorische Potenzial in queerer disidentification wie auch historisch spezifische Grenzen sexueller Politiken aufgezeigt werden.

\section{Spannungen im Material}

Der Song Manipulate, ${ }^{12}$ einer der ersten veröffentlichten Songs der Band aus dem Jahr 1992, zeigt das Spannungsverhältnis auf, in dem sich Tribe $8 \mathrm{zu}$ den Diskursen der lesbisch-feministischen Kultur bewegen. Entlang von Text und Gesangsweisen lassen sich einige Konflikte nachzeichnen. Im Intro des Stücks setzen die Musiker_innen zum gemeinsamen Gesang an:

\section{Women's love is so friendly \\ Women's love like herbal tea \\ Women's love it empowers me}

Ihre Stimmen klingen grölend, aggressiv, sie nerven und sind keineswegs freundlich-weich oder liebevoll, wie es der Songtext nahelegen könnte. Tatsächlich wird eher geschrien als gesungen. Im Text geht es um die ermächtigende
8 Unter dem Begriff «Re-Framing» verstehe ich in diesem Text - abgeleitet von engl. to reframe - die Neujustierung der Analyseperspektive auf die ästhetischen Politiken der Band.

9 Vgl. Rosemary Hennessy: Profit and Pleasure. Sexual Identities in Late Capitalism, New York 2018 [2000].

$10 \mathrm{Vgl}$. Woltersdorff: Queer Theory und Queer Politics, 915.

11 Vgl. José Esteban Muñoz: Disidentifications. Queers of Color and the Performance of Politics, Minneapolis 1999.

12 Manipulate, erschienen auf: Pig Bitch, USA 1992. 
13 Die Protagonist_innen der Women's Music kritisierten die Abwesenheit von positiven Images von Weiblichkeit in der populären Musik und fehlende Chancen für Frauen im Musikgeschäft. Nicht nur mit musikalischen Inhalten, auch durch den Aufbau unabhängiger Produktionsund Distributionsstrukturen wollten sie der patriarchalen Kultur, innerhalb derer Mainstream-Pop-Musik existierte, direkt entgegentreten. Siehe dazu: Martha Mockus: Music, Women's, in: Bonnie Zimmerman (Hg.): Lesbian Histories and Cultures. An Encyclopedia, New York, London 2012, 521-524, hier 522.

14 Vgl. ebd., 521.

15 Vgl. Reebee Garofalo: Rockin' the Boat. Mass Music and Mass Movements, Cambridge 1992, 244.

16 Vgl. Alice Echols: Cultural Feminism. Feminist Capitalism and the Anti-Pornography Movement, in: Social Text, Vol. 7, 1983, 34-53, hier 35 .

17 Vgl. Evelyn McDonnell: Queer Punk trifft Womyn's Music, in: Anette Baldauf, Katharina Baumgartner (Hg.): Lips, Tits, Hits, Power. Popkultur und Feminismus, Wien, Bozen 220-227; Katja Kailer, Anja Bierbaum: Girlism. Feminismus zwischen Subversion und Ausverkauf, Berlin 2002; Ann Cvetkovich: An Archive of Feelings. Trauma, Sexuality, and Lesbian Public Cultures, Durham, London 2003

$18 \mathrm{Vgl.} \mathrm{McDonnell:} \mathrm{Queer} \mathrm{Punk}$ trifft Womyn's Music, 226.

$19 \mathrm{Vgl}$. Beyer: Tribe 8, 287f.

20 Das ist eine Gürtelkonstruktion, mit deren Hilfe Dildos z. B. um die Hüfte geschnallt werden können.

$21 \mathrm{Vgl}$. Cvetkovich: An Archive of Feelings, 85 .

22 Vgl. ebd., 86.
Wirkung der Liebe unter Frauen. Begriffe wie «friendly» und «herbal tea» wirken klischeehaft, sind im US-amerikanischen Kontext der Band jedoch leicht als Anspielung auf lesbischen Separatismus und die damit häufig verbundene musikalische Tradition der Women's Music erkennbar. ${ }^{13}$ Diese bildete in den USA seit den I96oer Jahren den ersten popkulturellen Kontext, in dem offen über lesbisches Leben, Begehren und Sexualität gesungen wurde. ${ }^{14}$ Ihre Verfechterinnen setzten auf lesbischen und feministischen Separatismus, der zu einer enormen Zunahme der von Frauen gemachten Musik führte. ${ }^{15}$ Festivals wie das Michigan Womyn's Music Festival (MWMF) wurden mit der Absicht gegründet, einen safe space, einen sicheren Ort für die Musik von Frauen und die Entwicklung weiblicher Kultur zu schaffen. Doch während dadurch neue Entfaltungsmöglichkeiten und Erfahrungsräume entstanden, entwickelten sich auch neue Normen, die begrenzend wirkten. In der Suche nach dem radikalen Bruch mit den Mustern der hegemonial-patriarchalen Kultur wurden zum Teil dogmatische Vorstellungen davon entwickelt, was Frauen vermeintlich schadete und was sie ermächtigte. Entwürfe einer neuen Weiblichkeit reichten zum Teil so weit, dass den patriarchalen Strukturen die essenzialistische Vorstellung einer eigenen, besseren und weniger unterdrückerischen Sexualität gegenübergesetzt wurde, die andere Formen lesbischer Gender- und Sexualitätsentwürfe ausschloss. ${ }^{16}$

Ich lese die gesangliche Performance von Tribe 8 zu Beginn des Stückes als Intervention in die Kontroversen um Sexualität, die sich mit den Sex Wars deutlich zugespitzt hatten. Vor dem Hintergrund dieser Weiterentwicklungen muss auch der Auftritt der Band 1994 auf dem MWMF betrachtet werden. ${ }^{17}$ Einige Teilnehmer_innen hatten den Auftritt der Band durch Proteste verhindern wollen. Sie warnten davor, dass Tribe 8 Gewalt gegen Frauen propagiere. ${ }^{18}$

Besonders zwei Punkte hätten Anlass dafür gegeben: S/M-Elemente in der Show und Breedloves Dildo-Performance, die als Ritual zu jedem Tribe 8-Auftritt gehörte ${ }^{19}$ - während des Songs Frat Pig, in dem es um kollektive Rachebzw. Kastrationsfantasien nach einer Vergewaltigung geht, holte Breedlove einen Dildo, den er im strap-on ${ }^{\mathbf{2 0}}$ unter der Hose trug, heraus, schnitt ihn mit einem Messer ab und warf ihn in die Menge. Ann Cvetkovich beschreibt in ihrer inspirierenden Analyse, wie die Bandmitglieder auf die Vorwürfe reagierten, indem sich einige von ihnen selbst als Überlebende von Gewalt zu erkennen gaben und erklärten, wie ihnen die Musik und ihre Auftritte ermöglichten, einen Umgang mit Aggressionen und Schmerz zu finden. ${ }^{21}$ Im Workshop, den die Band nach dem Konzert anbot, gaben viele Besucher_innen an, ihre Meinung über die Performance geändert zu haben, als sie erkannten, dass die Band sexualisierte Gewalt adressiere, nicht promote. ${ }^{22}$

Das Festival bildet einen Knotenpunkt in der Organisation der lesbischfeministischen Kultur, die Tribe 8 in Manipulate thematisieren. Während Cvetkovich die transformatorischen Potenziale kollektiver Auseinandersetzungen um Traumata betont, lassen sich im Song durchaus auch Provokationen 
gegenüber Diskursen dieser Kultur ausmachen. Während der Text Assoziationen zu den Texten der Women's Music hervorruft, ${ }^{23}$ zeigt der grölende Gesang, dass sich die Band hier auch abgrenzen will. Jedoch beschreibt Breedlove, wie er selbst früher viel Women's Music gehört habe. In Manipulate mache er sich auch über sich selbst lustig:

Ich trinke viel Kräutertee [...] ich verehre die Göttin ... ich bin genauso wie die, über die ich mich da lustig mache. Wenn wir <Manipulate> ansagen, dann sagen wir vorher alle zusammen [mit weicher Stimme]: <Laßt uns zu einem Kreis der Heilung zusammentreten!> Die Leute sind beleidigt, weil sie denken, daß ich die anderen verarsche, aber das stimmt nicht. Ich vereine in mir all diese verschiedenen Widersprüche; das tun wir alle. ${ }^{24}$

Aus der Interviewpassage wird deutlich, dass sich die Textbotschaft nicht allein nach außen auf kritisierte lesbische Zusammenhänge und ihre Politiken richtet. Stattdessen richtet Breedlove den Blick auch auf sich selbst bzw. schreibt sich in dieses Kollektiv ein. Die Performance kann so als humorvolle Weise verstanden werden, sich selbst und anderen einen Spiegel vorzuhalten. Besonders heilsam wirkt dieser Gesang indes nicht. Im Gegenteil ist er disharmonisch, Töne werden verfehlt, Gesangslinien driften zum Teil auseinander. Er erzählt eine andere Geschichte als der Text. Daher gehe ich davon aus, dass das Konzept «Harmonie» als solches problematisiert wird. Auch die Vorstellung harmonisch-lesbischer Gemeinschaft wird hier unterlaufen, weil die Stimmen den Text durch die starke Betonung von Inkohärenzen und des Nichtharmonischen in einer Weise bearbeiten, die seine Aussagen unglaubwürdig erscheinen lassen. Ich lese das Zusammenspiel von Text und spezifisch entworfener Gesangsweise als Versuch einer Destabilisierung der Normen, die lesbische Gemeinschaftsund Identitätsvorstellungen prägen und die einige Entwürfe privilegieren, während sie andere abwerten.

Identitätsbildungen und Subjektivierung sind immer miteinander verwoben und kollektive Prozesse. Dies zeigt sich in (sub-)kulturellen Gemeinschaften, in denen bestimmte Identitätsanteile, die in der breiteren Öffentlichkeit ausgeblendet oder abgewertet werden, stärker leb- und verhandelbar werden. Das Zusammenspiel aus Text und Gesangsweise lässt sich mit einem Begriff von José Muñoz als Praxis der disidentification deuten. Er charakterisiert diese als strategische Weise der Selbstinszenierung, die sich hegemonial zur Verfügung stehenden Identifikationsangeboten weder komplett anpasst noch diese verwirft, sondern sie durch taktische, partielle Bezugnahmen quasi von innen heraus umarbeitet: ${ }^{25}$ Zwischen den Polen von Identifizierung und Gegenidentifikation ermöglicht Disidentifikation eine dritte, ausgehandelte Position, in der die Anrufungen des Subjekts neu verhandelt werden können. ${ }^{26}$ Im Rückgriff auf diese Strategie können die Musiker_innen Normalisierungen kritisieren und damit eine veränderte Position für sich innerhalb der kritisierten Szenen aushandeln.
23 Hier zwei Beispiele: Alix Dobkin veröffentlichte 1973 das bedeutende Album Lavender Jane Loves Women. Im Song View From Gay Head heißt es im Refrain: «Lesbian, living in no-man's land. Lesbian, lesbian, any woman can be a lesbian». Mit über 500 ooo verkauften Exemplaren wurde Cris Williamsons Album The Changer and the Changed (1975) zu einem der populärsten Alben der Women's Music. Der Song Sweet Woman darauf macht lesbisches Begehren explizit in Zeilen wie: "Sweet woman, risin" inside my glow, I think I'm missin' you, Singin' to me them soft words, Takin' me to your secret".

24 Andrea Juno: Angry Women. Die weibliche Seite der Avantgarde, St. Andrä-Wördern 1997 [1996], 77. 25 Vgl. Muñoz: Disidentifications, 120.

26 vgl. ebd., 83. 
Im weiteren Verlauf des Stücks übernimmt Breedlove allein den Gesang. Nun bricht der Text um, von freundlicher Frauenliebe zu expliziten BDSMFantasien, die voller Machtdynamiken sind:

\section{I just wanna manipulate my girlfriend, I just wanna play games with her head. I want her to do some mental push ups, I want ber to apologise and beg.}

Während das Begehren zum Ausdruck gebracht wird, die Partnerin zu manipulieren, zu kontrollieren, werden wiederkehrend Schuldgefühle beschrieben: «It's a sin, it's so wrong, I feel guilty as fuck», dann wieder offensiv zurückgewiesen: «I don't give a fuck what you think». Breedlove bezieht eine konfliktreiche und schuldbeladene Außenseiterposition, die er zum Teil offenbar jedoch auch gern einnimmt. Nahe liegt, den Text so zu deuten, dass er provozieren und durch die Benennung des «girlfriends» explizit lesbisch markierte BDSM-Praktiken verhandelbar machen möchte.

Explizite Repräsentationen von klassenbezogenen Themen sind in Tribe 8s ästhetischen Praktiken nicht erkennbar, wenn man diese als einfaches Abbild oder Ausdruck bereits geformter, einheitlicher Klasseninteressen und -identitäten begreifen will. Die britischen Cultural Studies haben aufgezeigt, dass ein solches Verständnis reduktionistisch ist. Die in dieser Denktradition entstandenen Analysen zu Klasse und Kultur bieten - wie ich im Folgenden aufzeige - Anknüpfungspunkte, reichen aber nicht aus, um im Rahmen eines Re-Framing das komplexe Verhältnis queer-feministischer ästhetischer Praktiken zur $<$ Klassenfrage $>$ neu zu lesen.

\section{(Des-)Artikulation von Klasse mit Differenz. Grenzen der Cultural Studies}

Die britischen Cultural Studies brachen mit dem bis dato im Marxismus verbreiteten Modell des Verhältnisses von ästhetischer Praxis und Klassenidentität. Anknüpfend an die Hegemonie-Theorie Antonio Gramscis analysierten sie, wie verschiedene gesellschaftliche Gruppen und Klassenfraktionen permanent um Richtung, Werte und Normalität des gesellschaftlichen Lebens ringen. Diese Kämpfe um Hegemonie, um Gestaltungsmacht, die sie (auch) als Klassenkämpfe verstehen, werden in den kulturellen, symbolischen und politischen Formen des sogenannten Überbaus ausgetragen. ${ }^{27}$ Für die britischen Forscher_innen ist Kultur immer (auch) ein Aushandlungsfeld konfligierender Klasseninteressen. Frühe Studien der Cultural Studies gingen häufig dennoch von einem Klassenverständnis aus, das weniger intersektional ausgerichtet war. Seit etwa I 975 zeichnete sich eine Wende im Denken über das Verhältnis von Klasse, Identität und Differenz ab und darüber, wie Kämpfe um Hegemonie entsprechend interpretiert werden können. Zunehmend wird in den Arbeiten 

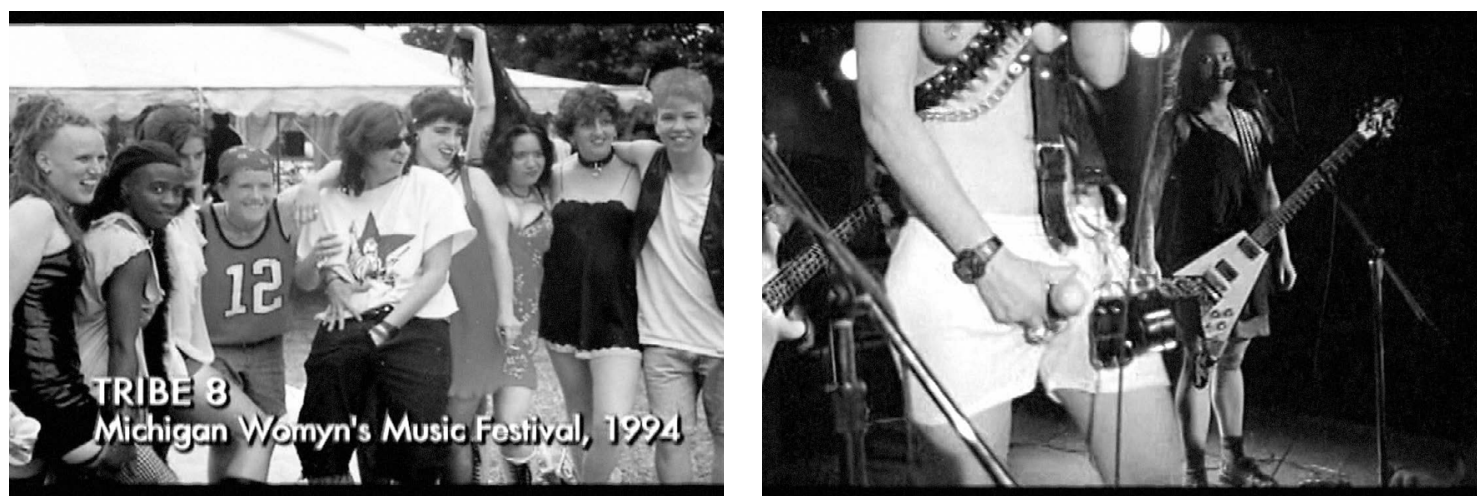

erkennbar, dass in den vielfältigen und dezentralen gesellschaftlichen Auseinandersetzungen um Hegemonie keineswegs der Bezug auf eine Klassenidentität im Mittelpunkt stehen muss: Vielmehr artikulieren sich in unterschiedlichen gesellschaftlichen Situationen die Konflikte sehr verschieden und werden durch andere Auseinandersetzungen, etwa um Rassismus, Sicherheit oder Religion, überlagert. ${ }^{28}$

Die Ansätze verdeutlichen, wie hegemoniale Herrschaft gerade auf der relativen Unsichtbarkeit, Einhegung und Verschiebung von Klassenkonflikten beruht. Im Nachkriegsgroßbritannien hatten steigende Löhne und ein vom Sozialstaat gespanntes Sicherheitsnetz die Lebensweise größerer Teile der Arbeiterklasse verändert. Dies führte zu der Sichtweise, dass der Klassenkonflikt vermeintlich beigelegt, die Arbeiterklasse magisch verschwunden sei. Der neu entstehende Sozialtyp der Jugend(-lichen) wurde medial zur vermeintlich klassenlosen Metapher <sozialen Wandels> (v)erklärt ${ }^{29}$ und die spektakulären Subkulturen als Ausdruck eines Generationenkonflikts gedeutet. Die Cultural Studies betonten dagegen die Herkunft von Teds, Mods und später der Punks aus der Arbeiter_innenkultur. Subkulturen wurden in den Untersuchungen als Widerstandsformen begriffen, mithilfe derer ihre Mitglieder symbolische Kämpfe um Hegemonie austrugen. Die Jugendlichen suchten darin Antworten «to the problems posed by a framework of bourgeois institutions but that response is the response from a working class experience of those institutions». ${ }^{30}$

Dick Hebdige präzisierte die Bedeutung der Stilpraktiken, mit der die Jugendlichen gegen ihre Klassen- und andere Weisen der Unterdrückung rebellierten. ${ }^{31}$ Schon früh kritisierten Feminist_innen wie Angela McRobbie und Jenny Garber die Ausblendung der Geschlechterdifferenz in den Studien und zeigten das enge Verständnis von Klassenidentität als männlich (und, wie hier zu ergänzen ist, als weiß und heterosexuell) auf. In den Untersuchungen zur Kultur von Arbeitermädchen rückte die häusliche Sphäre und die Bildung von
Abb. $1 / 2$ Tribe 8 auf dem Womyn's Music Festival. Konzert mit Dildo-Performance von Lynnee Breedlove. Screenshots aus: Rise Above. The Tribe 8 Documentary, Regie: Tracy Flannigan, USA 2003

28 Vgl. Stuart Hall: Ideologie und Ökonomie: Marxismus ohne Gewähr, in: ders.: Ideologie, Identität, Repräsentation. Ausgewählte Schriften, Bd. 4, hg. v. Juha Koivisto u. Andrea Merkens, Hamburg 2004 [1983], 8-33.

29 Vgl. Hall u. a.: Subcultures, Cultures, and Class, 9, 18.

30 Paul Corrigan, Simon Frith: The Politics of Youth Culture, in: Stuart Hall, Tony Jefferson: Resistance Through Rituals, London 1993 [1975], 195-204, hier 199.

31 Vgl. Hebdige: Subculture. 
$32 \mathrm{Vgl}$. Angela McRobbie, Jenny Garber: Girls and Subcultures. An Exploration, in: Hall u.a. (Hg.): Resistance Through Rituals, 209-222. 33 Vgl. Raymond Williams: Culture is Ordinary, in: Jim McGuigan (Hg.): Raymond Williams on Culture and Society. Essential Writings, London 2014 [1968], 1-18.

34 Vgl. Judith (Jack) Halberstam: In A Queer Time and Place. Transgender Bodies, Subcultural Lives, New York 2005, 161.

35 Vgl. Linda Garber: Identity Poetics. Race, Class and the LesbianFeminist Roots of Queer Theory, New York 2001, 99 f.; Keenga-Yamahtta Taylor: How We Get Free. Black Feminism and the Combahee River Collective, Chicago 2017, 8f.

36 Vgl. Lisa Duggan: The Twilight of Equality? Neoliberalism, Cultural Politics, and the Attack on Democracy, Boston 2003.

37 Vgl. Wendy Brown: Wounded Attachments, in: Political Theory, Vol. 21, Nr. 3, 1993, 390-410.

38 Vgl. ebd., 394 .

39 Ebd., 395.

40 vgl. ebd., $394 f$.

41 Ebd.
Fankulturen in den Fokus. ${ }^{32}$ Wurde Kultur von Williams als gesamte Lebensweise, als «whole way of life» ${ }^{33}$ begriffen, machten die feministischen Untersuchungen eine klassenspezifische Lebensweise zugleich als geschlechtsspezifisch geformt verstehbar. Differenzen unter Mädchen werden jedoch auch hier ausgeblendet, etwa durch den implizit heteronormativen Fokus der Studien. ${ }^{34}$

Arbeiter_innen- und Mittelklassekulturen wurden im Rahmen der Cultural Studies insgesamt als je homogen konzipiert. Besonders problematisch ist die Zuordnung von gay subculture zur Mittelklasse, die Klassendifferenzen innerhalb dieser Gruppe unsichtbar macht. Die Analysen prägte eine eigentümliche Widersprüchlichkeit: Während kulturelle Praktiken als komplexe Artikulation von Klassenkonflikten wahrnehmbar wurden, blieb zugleich außen vor, welche Kämpfe um Hegemonie, um das Verhältnis von Klasse und Differenz innerbalb dieser kulturellen Felder stattfinden.

In anderer Weise wird dieses Verhältnis spätestens seit den I96oern im US-amerikanischen Kontext diskutiert. Wichtige Beiträge lieferten hier besonders schwarze Feminist_innen wie Angela Davis, bell hooks oder auch die Autor_innen des Combahee River Collective. Letztere prägten den Begriff der «identity politics»: Die Autor_innen betonten die Notwendigkeit, die eigene Identität gegen eine feindliche, sie auslöschende Umgebung sichtbar zu machen, und zugleich wandten sie sich gegen ein essenzialistisches Verständnis. Sie zeigten dagegen das umkämpfte Spannungsverhältnis von Fremdzuschreibungen und dem durch Mehrfachzugehörigkeiten und -positionierungen in sich überlagernden Macht- und Herrschaftsverhältnissen geprägten Selbst auf. ${ }^{35}$

Aus einer kritischen queeren Perspektive legt Lisa Duggan problematische Effekte der zunehmenden Institutionalisierung und Professionalisierung von Teilen der identitätspolitischen Bewegungen dar. ${ }^{36}$ Zugleich wertet sie die in einem anderen US-Diskurs - dem der liberalismuskritischen Linken - häufig anzutreffende Gegenüberstellung von Klassen- vs. Identitätspolitiken als zu pauschal. In diesen Debatten, die sich eher auf einer Metaebene der Kritik an Identitätspolitik nähern, finden trotz des anderen zeit-räumlichen Kontextes ähnliche Grenzziehungen statt wie in den britischen Cultural Studies. So geht etwa Wendy Brown mit Identitätspolitiken des Feminismus und der gay liberation hart ins Gericht: ${ }^{37}$ Die politische Durchschlagskraft US-amerikanischer Identitätspolitiken seit den I970er Jahren sei mit einer Re-Naturalisierung von Kapitalismus verbunden. ${ }^{38}$ Die «invisibility and inarticulatedness of class ${ }^{39}$ sei kein historischer Unfall, sondern konstitutives Moment der Identitätspolitiken, die dem liberalen Diskurs verhaftet blieben und die durch kapitalistische Gewalt, Rassismus und Patriarchat erlittenen Verletzungen am normativen Ideal der Mittelklasse messen..$^{40}$ In diesem Sinne repräsentiere

[middle class] the normalization rather than the politicization of capitalism, the denial of capitalism's power effects in the ordering of social life, the representation of the ideal capitalism to provide the good life for all $[\ldots] .^{41}$ 
Diese Kritik an Politiken, die auf Inklusion, Gleichberechtigung und staatliche Anerkennung pluraler Identitäten ausgerichtet sind, leuchtet ein. Aber nicht alle Identitätspolitiken orientieren sich am Mittelklasseideal, wie z.B. die bereits genannten Positionierungen und Debattenbeiträge schwarzer Feminist_innen zeigen.

Duggan prägte den Begriff der «Homonormativität», ${ }^{42}$ um damit in den I990er Jahren in schwul-lesbischen Bewegungen entstehende Politiken zu beschreiben, die mit ihren Forderungen, u. a. nach der Homoehe, auf eine privatisierte, depolitisierte, in Domestizität und Konsum verankerte schwul-lesbische Kultur zielten und Fragen nach Verteilungsgerechtigkeit ausblendeten. ${ }^{43}$ Sie fordert eine stärkere Differenzierung zwischen solchen normalisierenden Ansätzen und radikalen antirassistischen, queeren, feministischen Ansätzen, deren Umverteilungskämpfe auf Redistribution von Ressourcen $<$ nach unten $>$ zielten.

Eine Perspektivierung von Kultur als gesamter Lebensweise, die Identität, Klasse und Differenz in ihrer Verschränkung analysiert, müsste - anders als die früheren Cultural Studies und ohne die Dichotomie von Klassen- vs. liberale Identitätspolitiken zu reproduzieren - aufzeigen, durch welche Konfliktdynamiken Klasse historisch spezifisch artikuliert und überlagert wird, welche komplexen und mehrfachen Identitäten und Disidentifikationen sich in diesen Auseinandersetzungen bilden. Es geht kurz gesagt darum, «Klasse mit Differenz» zu denken.

\section{Re-Framing part I: butch/femme-Dynamiken}

Butch/femme-Dynamiken und sexuelles Power-Play gaben innerhalb der (lesbisch-)feministischen Bewegung wiederholt Anlass zu Diskussionen um Klasse und lesbische Identität.

Leslie Feinbergs Klassiker Stone Butch Blues machte lesbische Lebensweisen und heterogene Genderentwürfe in der Arbeiter_innenklasse einer breiteren Leser_innenschaft zugänglich. ${ }^{44}$ Für lesbische Arbeiter_innen waren die Bars und Privatpartys wichtige Orte, um andere Frauen treffen und Lieb- oder Freundschaften mit ihnen knüpfen zu können. ${ }^{45}$ Der Bezug auf die Genderrollen butch und femme hatte in diesen lesbischen working-class communities eine bedeutende Rolle. ${ }^{46}$ Das Spiel damit machte es möglich, sich in der Öffentlichkeit stärker wahrnehmen zu können, und ging mit der Entwicklung spezifischer Begehrenspraktiken einher. ${ }^{47}$ Auf diese <lesbische Ahnengeneration>, die zum Teil als politischer Vorläufer der modernen LGBTIQ-Bewegung gilt, ${ }^{48}$ beziehen sich Tribe 8 in ihren Entwürfen und machen sie - wieder - zum Ausgangspunkt, um Fragen der Sexualität zu thematisieren. Denn in den I980er Jahren warfen Cherrie Moraga und Amber Hollibaugh der feministischen Bewegung vor, Lesbischsein vor allem als politisches bzw. intellektuelles Konzept zu fassen. Umschifft würde die Frage der lesbischen Sexualität, insbesondere die Machtdynamiken in sexuellen Praktiken von butches und femmes. ${ }^{49}$ Dadurch würden die Erfahrungen ganzer
42 Vgl. Lisa Duggan: The Twilight of Equality? Neoliberalism, Cultural Politics, and the Attack on Democracy, Boston 2003, 65 .

43 Vgl. ebd. Den zunehmenden Normalisierungskurs beschreibt auch Woltersdorff. Er sei gekennzeichnet durch die zunehmende Institutionalisierung der politischen Bewegung der Schwulen und Lesben, die lobbypolitische Orientierung und die Verengung ihrer Repräsentationen, «die stillschweigend ihre weißen, mittelständischen und männlichen Vertreter zur Norm machte». Woltersdorff: Queer Theory und Queer Politics, 914.

$44 \mathrm{Vgl}$. Leslie Feinberg: Stone Butch Blues, Los Angeles 1993.

$45 \mathrm{Vgl}$. Elizabeth Lapovsky Kennedy, Madeline D. Davis: Boots of Leather, Slippers of Gold. The History of a Lesbian Community, London 2014 [1993], 5.

46 Die Entwürfe von Lesben aus der Mittelklasse waren dagegen häufig diskreter, u. a. auch deswegen, weil sie Angst hatten, ihre Karrieren zu verlieren, würde ihre Homosexualität entdeckt. Vgl. Lillian Faderman: Odd Girls and Twilight Lovers. A History of Lesbian Life in Twentieth-Century America, New York 1991, $473 \mathrm{ff}$.

47 Vgl. Kennedy u. a.: Boots of Leather, Slippers of Gold, 5 .

48 Vgl. ebd., 1.

49 Vgl. Hollibaugh u. a.: What We're Rollin Around in Bed With. Sexual Silences in Feminism: A Conversation toward Ending Them, in: Heresies. A Feminist Publication on Art and Politics, Vol. 3, 1981, 58-62, hier 59 . 
Communitys abgewertet. Ihr race- und Arbeiter_innenklassenhintergrund, der maßgeblich ihre Identifikationen mit diesen Rollen und Dynamiken beeinflusst, habe aber große Bedeutung für ihr politisches Selbstverständnis. ${ }^{\mathbf{5 0}}$

Während also vordergründig sexuelle Praktiken und lesbische Geschlechterentwürfe den Streitpunkt innerhalb der feministischen Bewegung bilden, lassen sich diese Streite aus einer anderen Perspektive auch als rigide und an Mittelklasse-Lebensweisen orientierte Fehlkonzeption eines einheitlichen feministischen Subjekts betrachten.

Vor diesem Hintergrund kann das Zusammenspiel aus Text und Gesang in Manipulate neu gelesen werden: Die schon angesprochene disidentifikatorische Strategie ermöglicht es den Musiker_innen, in einheitliche Subjektvorstellungen zu intervenieren. Damit ist auch verbunden, Klassenerfahrungen, die in lesbisch-feministischen Bewegungen an den Rand gedrängt wurden, wieder mit in den Fokus zu rücken.

In einzelnen Songs treten die Klassendynamiken in den Auseinandersetzungen um butch/femme-Dynamiken expliziter hervor: Der Song Neanderthal $D y k e^{51}$ greift zunächst Diskussionen um patriarchale Schönheitsstandards auf, deren Erfüllung häufig sehr femininen Lesben (femmes) zum Vorwurf gemacht wurde: «Patriarchal standards of beauty is what my pc girlfriend will have me refuting». Als $p c$ - also politisch korrektes - girlfriend wird in zugegebenermaßen stereotypisierender Weise die Figur der verurteilenden Lesbe wahrnehmbar, die ein Problem mit den Entwürfen hat. Dann jedoch rücken an Bildungsprivilegien geknüpfte Klassendifferenzen in den Fokus: «Neanderthal Dyke, Neanderthal Dyke, Never read Dworkin, I ride a big bike, Feminist theory gets me uptight, Get in some heels and lipstick and I'll spend the night». Die US-amerikanische Feministin Andrea Dworkin, auf die hier angespielt wird, war eine der populärsten Pornografie-Gegnerinnen und polarisierte innerhalb der feministischen Sex Wars. Ich lese sie hier als Sinnbild für jene Diskurse innerhalb des lesbischen Feminismus, die Tribe 8 kritisieren. Wendungen wie «I ride a big bike» oder Begriffe wie lipstick und heels codieren in Neanderthal Dyke die Identitäten von butch und femme; spezifischer noch werden Bezüge zwischen butch-Identität und der cis-männlichen, maskulinen Figur des Bikers - und damit einer historischen Subkultur, deren Basis ebenfalls junge Arbeiter bildeten - hergestellt. Der Begriff Neanderthal Dyke selbst macht in zweifacher Hinsicht auf die dadurch <uralt> wirkenden lesbischen Entwürfe butch und femme aufmerksam. Einerseits würdigt er ihre lange Geschichte, andererseits tauchen sie als das Ausgemusterte, scheinbar andere populärer, auch theoretischer Debatten auf, die maßgeblich Zielrichtungen und Gesellschaftsverständnis des Feminismus der Zeit formten. Der Text kritisiert damit auch die in diesem Zusammenhang wirkmächtigen Bildungsprivilegien, die Zugang

50 Vgl. Hollibaugh u. a.: What We're Rollin Around in Bed With, 62. 51 Neanderthal Dyke, erschienen auf: Pig Bitch, USA 1992. zu Deutungs- und Gestaltungsmacht des-/organisieren. Butch- und femmeIdentitäten und sexuelle Dynamiken werden so zum Ausgangspunkt gemacht, um Konflikte zuzuspitzen: 
«My political consciousness is fried, I'm not exactly woman identified», singt Breedlove. Hervorgehoben wird im weiteren Song sowohl, dass nicht alle dieselben intellektuellen Voraussetzungen haben, um an bestimmten feministischen Diskursen überhaupt teilnehmen zu können, als auch die Tatsache, dass die darin ausgehandelten impliziten Normen von akzeptablen und nichtakzeptablen Genderentwürfen letztlich Mittelklassenormen sind. Die Abgrenzungsfolie einer intellektualisierten lesbischen Mittelklasse, der die prollige Inszenierung als motorradfahrende, sexuell direkte butch in schematischer Weise entgegengesetzt wird, reproduziert Klischees und ist ambivalent. In der rotzigen Formulierung «Pseudo-intellectual slut, you went to school, did you learn how to fuck?» wird eine anti-intellektuelle Haltung deutlich. Das Mittel textlicher Provokation wird zugleich ausgereizt, um die Kombination aus Mittelklassedistinktion und Abwertung nichtkonformer Sexualität in drastischer Weise zurückzuweisen. Komplizierter noch wird es dadurch, dass in Subkulturen Zeichen, die vermeintliche workingclassness codieren, auch unabhängig von ökonomischer Herkunft zur Selbststilisierung als z. B. authentisch oder rebellisch eingesetzt werden können. Dabei ist aber zu berücksichtigen, dass sich Tribe 8 eben nicht linear auf männliche Arbeiterklasse beziehen, sondern sich auch in Beziehung zur Tradition der dykes on bikes, lesbischer Biker_innen, setzen.

\section{Part II: Sexualität und Trauma - Gender und class in Tribe 8s Politik der Gefühle}

Durch das Aufbrechen einheitlicher Subjektvorstellungen kann die Band dazu beitragen, Erfahrungen der Differenz im Spannungsfeld von Feminismus, Lesbischsein und Klasse zu artikulieren. Dies ist aber nur dadurch möglich, dass sie ihren Platz als Teil der lesbisch-feministischen Bewegung nicht aufgibt.

Viele Arbeiten der Band beziehen sich auf die gewaltvollen Strukturen, die weibliche Sexualität prägen. Cvetkovich positioniert Tribe 8 als Teil lesbischer öffentlicher Kulturen, die Sexualität und Paarintimität aus dem Schlafzimmer holten und sie, gerade ohne die Gefahren von Sexualität zu ignorieren, zum Fokus kollektiver Auseinandersetzungen um BDSM-Praktiken, Dildogebrauch oder butch/femme-Dynamiken machten. ${ }^{52}$

Die Praktiken von Tribe 8 ermöglichen, wie bereits diskutiert, spezifische Zugänge zu Traumata. Sie verwehren sich dagegen, Überlebende sexualisierter Gewalt als Opfer darzustellen. Die öffentlichen Kulturen, die sich um lesbische Sexualität geformt haben, werden als Ressource für wütende und humorvolle Antworten auf Traumata genutzt. ${ }^{53}$ Die Performancepraktiken und Inhalte der Texte attackieren die Trennung von öffentlich und privat und begehren gegen die damit verbundenen Machtverhältnisse auf. Im Workshop, in dem nach dem Konzert nochmal <über alles geredet〉 werden kann, werden Perspektiven und Erfahrungen kollektiv verhandelt und geteilt. Dass der wütende Zugang der Band zunächst auf so starke Abwehr traf, zeigt - neben der Wirkmächtigkeit

52 Vgl. Cvetkovich: An Archive of Feelings, 4.

53 Vgl. ebd., 13. 
ihrer Provokation - auch die Grenzen <legitimer> Auseinandersetzungsformen in der feministischen Bewegung auf, die dazu beitragen, eine Hegemonie weißer, gebildeter Feminist_innen ${ }^{54}$ zu sichern.

Für Menschen, die keinen Zugang zu den in den USA oft teuren Therapien haben, können die subkulturellen Öffentlichkeiten, die um Traumata entstehen und die Zirkulation von Wissen ermöglichen, eine wichtige Ressource sein. So gibt Breedlove im Workshop u.a. Tips, wie Dildos kostengünstig besorgt und für kathartische, heilsame Zwecke eingesetzt werden können. ${ }^{55}$ Die Etablierung einer subkulturellen Öffentlichkeit lässt sich in diesem Sinne als eine Politik kollektiver Umverteilung von Wissen und Zugang zu Ressourcen verstehen, die Selbstermächtigung nicht an ökonomische Privilegien knüpft. Dabei ist jedoch kritisch zu reflektieren, dass Öffentlichkeit selbst, auch in subkultureller, gegen-hegemonialer Form, kein machtfreier, sondern von Klassenverhältnissen durchzogener Raum ist. Die Entstehung lesbisch-feministischer Öffentlichkeiten ist auch an das Werteverständnis derer geknüpft, die sie produzieren. Habitus, Bildungsprivilegien, Form und Inhalt von Aussagen und Gesten prägen, wer sich im öffentlichen Ort des Workshops selbstverständlicher wohlfühlt oder daran überhaupt teilnehmen mag. Über die genauen Machteffekte kann an dieser Stelle nur spekuliert werden. Fest steht, dass an dem Festival Menschen mit unterschiedlicher Klassenpositionierung teilnahmen und Teilnehmer_innen den Workshop als ermächtigend beschrieben.

\section{Part III: queere Politiken der Wut und Allianzen}

Tribe 8s spezifische Aneignung des sexpositiven Ansatzes und die Re-Artikulation von butch- und femme-Identitäten bzw. -Dynamiken sind nur vor dem Hintergrund neuer queerer Politiken der Wut im Kontext der Aidskrise begreifbar. Bis I 990 waren bereits mehr als I00.000 Menschen an Aids gestorben, auch da nicht genügend staatliche Gelder in die Erforschung der Krankheit und Gegenmaßnahmen investiert wurden. Die Reagan-Administration weigerte sich, Aids und das Ansteckungsrisiko mit HIV als gesamtgesellschaftliches Problem anzuerkennen. Als gefährdet wurden allein ohnehin marginalisierte Randgrup-

54 Vgl. Audre Lorde: The Uses of Anger. Women Responding to Racism, in: dies.: Sister Outsider. Essays and Speeches, Berkeley 2007 [1981], 83-88, hier 83.

55 Vgl. Cvetkovich: An Archive of Feelings, 86.

56 Woltersdorff: Queer Theory und Queer Politics, 914.

57 Vgl. ebd.

58 Vgl. Cvetkovich: An Archive of Feelings, 5 .

59 Auch Howard bevorzugt mittlerweile das männliche Pronomen. pen dargestellt: «Schwarze, Schwule, Prostituierte, Junkies». ${ }^{56}$ Ihnen wurde vorgeworfen, durch vermeintlich unverantwortliche Lebensführung selbst Verantwortung und Schuld für eine mögliche Erkrankung zu tragen. ${ }^{57}$

Die Aidskrise verdeutlichte, dass manchen Leben mehr Wert beigemessen wurde als anderen. Sie zeigte auch, wie Homophobie und Rassismus mitbestimmten, wessen Leid und Traumata zählten und öffentlich anerkannt wurden oder nicht. ${ }^{58}$ Ohnmacht angesichts der politischen Nichthandhabung der Krise motivierte maßgeblich die Wut im ästhetischen Ausdruck der Band. Das spiegelt sich exemplarisch in der Interviewaussage des Tribe 8-Gitarristen Silas Howard: ${ }^{59}$ 
[I]n that age, that era, anyone over a certain age in that scene was a rare thing, because so many people died. [...] It was this mind-blowing thing that we were in the wake of. I feel like that informed all of our activism and urgency. ${ }^{60}$

In diesem Kontext wurde der Begriff «queer» angeeignet, um neue politische Identitäten zu entwerfen. ${ }^{61}$ Mit spektakulär inszenierten lesbisch-sexuellen BDSM-Praktiken rückte die Band explizit Formen von Sexualität in die Öffentlichkeit, die (der <christlichen Mehrheit $>$ ) als pervers galten. In ihren ästhetischen Strategien setzten Tribe 8 somit auf Konfrontationen, wie sie seinerzeit in den neu entstehenden QueerAktivismen häufiger zu beobachten sind, etwa in den Praktiken von
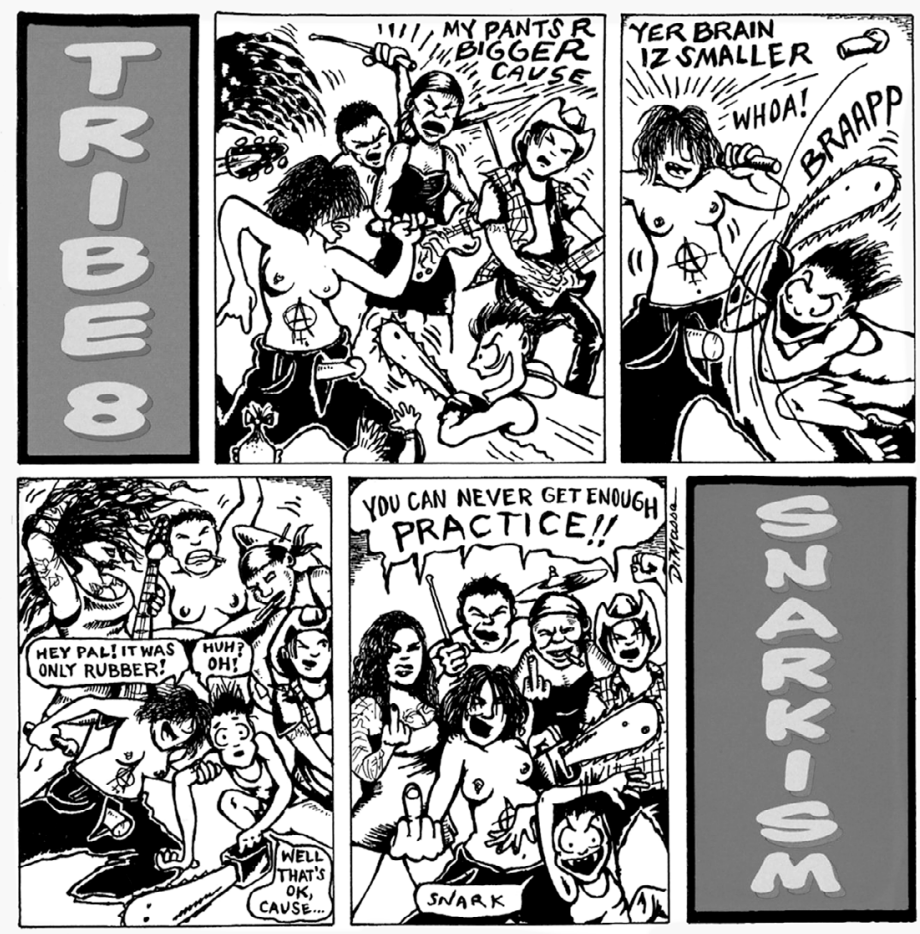
Gruppen wie Queer Nation.

Stereotype, negative Zuschreibungen an <perverse Homosexualität>, aber auch an <wütende Feminist_innen> werden von Tribe 8 nicht zurückgewiesen, sondern affirmiert, angeeignet und ausdrücklich zum Ausgangspunkt politischer (Selbst-)Entwürfe gemacht: Queer und der konfrontative Feminismus der Band sollen als Gefahr für die homophobe Politik und liberale Assimilation wirkmächtig werden und zugleich die in sexistischer, heteronormativer Alltagskultur virulente Zuschreibung der Machtlosigkeit an Frauen angreifen. Die Strategien zielen darauf ab, passivierende Gefühle der Scham und des Leids in Wut und Handlungsfähigkeit zu wenden.

Im Rückblick erscheinen die homophoben Politiken im Kontext der Aidskrise und liberale schwule und lesbische Identitätspolitiken als zwei Seiten einer «Nekropolitik», als Politiken des 〈Sterben-Machens», «for those who are unassimilable into liberal regimes of rights and representation and thus become disposable». ${ }^{62}$ Diese Nekropolitik hat mit dem Abbau des Wohlfahrtsstaats, mit fehlender Gesundheitsversorgung und der Repression marginalisierter Communitys eine starke rassisierte Klassendimension. Mit der Clinton-Administration vollzieht sich, was Nancy Fraser retrospektiv als Übergang zum «progressiven Neoliberalismus» ${ }^{63}$ bezeichnet: Schwul-lesbische Forderungen nach gesellschaftlicher Anerkennung und mehr Rechten wirken nun zunehmend erfolgreich, sie haben sich aber auf einen formalen Begriff von Gleichstellung verengt. Die Frage sozialer Gerechtigkeit bleibt außen vor. Die queeren

Abb. 3 Cover des Albums Snarkism der Band Tribe 8, USA 1996

60 Eric Torres: Q\&A: Silas Howard on the Unthinkable Bambi Lake and San Francisco's History of Fringe, Queer Art, in: Pitchfork, dort datiert 23.9.2015, pitchfork.com/thepitch/908qa-silas-howard-on-the-unsinkablebambi-lake-and-san-franciscos-historyof-fringe-queer-art|, gesehen am 20.2.2018.

$61 \mathrm{Vgl}$. Annamarie Jagose: Queer Theory. Eine Einführung, Berlin 2001, $123 \mathrm{f}$.

62 Jin Haritaworn, Adi Kuntsman, Silvia Posocco (Hg.): Queer Necropolitics, New York 2014, $1 \mathrm{f}$.

$63 \mathrm{Vgl}$. Nancy Fraser: Für eine neue Linke oder: Das Ende des progressiven Neoliberalismus, in: Blätter für deutsche und internationale Politik, Nr. 2, 2017, 71-76. 
Politiken der Wut zeichnet dagegen aus, dass sie Anerkennung von Leid und Gewalterfahrungen nicht von der Forderung nach einer Umverteilung von Ressourcen und Macht zugunsten derer trennten, denen Zugang dazu in der herrschenden Klassenordnung verwehrt bleibt. ${ }^{64}$

Anstatt die Anerkennung von Leid oder die Integration in staatliche Apparaturen zu fordern, stellen Tribe 8 in Form ästhetischer Mikropolitiken Fragen nach der gesellschaftlichen Verteilung von Macht ins Zentrum und diskutieren nicht allein Hegemonien außerhalb, sondern gerade auch innerhalb lesbischer, feministischer und LGBTIQ-Bewegungen. Statt die wenig produktive Trennung in Klassen- und Identitätspolitiken zu reproduzieren, lassen sich die ästhetischen Praktiken von Tribe 8 so auch als Ausdruck von Deutungskämpfen verstehen: darum, was feministische Politiken sein und wen sie ansprechen bzw. mitdenken könnten und sollten.

\section{Utopisches Potenzial und Grenzen in Tribe 8s kulturellen Politiken}

Das Re-Framing hin zu Klasse mit queerer Differenz ermöglicht es aber auch, die Grenzen dieser Politiken, deren Ausgangspunkt die eigene Erfahrung und Positionierung in den Auseinandersetzungen um Sexualität ist, zu diskutieren. Auch Tribe 8 stoßen an <identitätspolitische Grenzen>: Lesbisch-feministische Klassenerfahrungen mit Differenz werden sichtbar und zuvor vorrangig lesbische Allianzpolitiken erweitert. Die differenten Erfahrungen, die aus den spezifischen Verschränkungen von gender- und class- mit race-Zugehörigkeiten resultieren, werden dagegen weniger explizit thematisiert. Die Frage der Aushandlungen von Hegemonie wird nicht völlig bis in die unterschiedlichen Erfahrungen der Bandmitglieder mit Klasse und Rassismus hin zugespitzt. Auch der sexradikale Diskurs, innerhalb dessen die Band sich bewegt, muss kritisch reflektiert werden.

Denn der Bezug auf BDSM-Praktiken allein macht keine queere Klassenpolitik aus. Im Gegenteil kann darin implizit das Verständnis eines <freien Individuums> als Agent_in sozialer Veränderung und damit gerade ein bürgerliches Subjektverständnis reproduziert werden. ${ }^{65}$ Welche Privilegien verknüpfen sich damit, queere Sexualität feiern zu können? Rosemary Hennessy richtet den Blick auf den globalisierten Zusammenhang von Klassenverhältnissen und die gesellschaftliche Arbeitsteilung, durch die spezifische Lebensweisen und damit Formen des Lebens von Sexualität möglich werden. Queere Sexualität ist Teil dieser herrschaftsförmigen, kapitalistischen Arbeitsteilung, die weder ohne noch allein durch queer-feministische Allianzpolitiken überwunden werden kann.66

64 Vgl. Duggan: The Twilight of Equality?, xii.

65 Vgl. Hennessy: Profit and Pleasure, 181.

66 Vgl. ebd.
Das Re-Framing der Praxis von Tribe 8 legt so Widersprüche offen und im Rückblick auch die Frage nach spezifischen Konjunkturen queerer Allianzpolitiken nahe. Vor dem Hintergrund des progressiven Neoliberalismus, dem auch Teile der LGBTIQ- wie der Frauenbewegung zugerechnet werden müssen, 
kann der Blick auf Tribe 8 und die queeren Politiken der Wut heute in einer veränderten politischen Konjunktur verschärfter sozialer Ungleichheit und des erstarkten Rechtspopulismus dazu inspirieren, Klassenkonflikte innerhalb sozialer Bewegungen und der Queercommunity zuzuspitzen. ${ }^{67}$ Das Re-Framing kann dazu beitragen, Tribe $8 \mathrm{~s}$ in Auseinandersetzung mit lesbischer und feministischer Kultur ermächtigenden Umgang mit Trauma, Leid und Scham im Kontext heutiger Debatten um Klasse und «Scham» neu zu lesen. ${ }^{68}$ Volker Woltersdorff plädiert in kritischer Auseinandersetzung mit Didier Eribon dafür, dass das Sprechen über Scham eine «Triebfeder für eine intersektionale Klassenpolitik» sein könnte. ${ }^{69}$ Kulturelle und ästhetische Politiken können dazu beitragen, unterschiedliche Erfahrungen mit Gewalt, Ausschlüssen, Marginalisierung und Ausbeutung repräsentierbar und damit kollektiv reflektierbar zu machen. Sie könnten im besten Fall dabei helfen, Zugehörigkeiten und Differenzen und damit neue Allianzen marginalisierter Gruppen und innerhalb der vielfach fragmentierten Arbeiter_innenklasse vorstellbar zu machen, um Solidarität zu entwickeln - über unterschiedliche Erfahrungen und Positionierungen hinweg.
67 Vgl. Volker Woltersdorff: Für eine queerfeministische Klassenpolitik der Scham, in: Luxemburg. Gesellschaftsanalyse und linke Praxis, Spezialausgabe: Neue Klassenpolitik, 2017, 54-61, hier 58.

68 Vgl. Didier Eribon: Rückkehr nach Reims, Frankfurt/M. 2016; Didier Eribon: Gesellschaft als Urteil. Klassen, Identitäten, Wege, Berlin 2017.

69 Woltersdorff: Für eine queerfeministische Klassenpolitik der Scham, Go. 